\title{
The Harm of Fog and Thick Haze to the Body and the Prevention of Chinese Medicine
}

\author{
Le KANG, Yan-yan MIAO, Ming-san MIAO* and Zhen-ya HU \\ Henan University of Traditional Chinese Medicine, Zhengzhou, Henan, China, \\ 450046 \\ ${ }^{*}$ Corresponding author
}

Keywords: Fog and thick haze, Harm, Chinese medicine.

\begin{abstract}
In recent years, The situation that China's fog and thick haze pollution is very grim, which has threatened people's health and daily life work. fog and thick haze is a meteorological phenomenon, which is the essence of human activities caused by fine particulate pollution. With the increase in the severity of the fog and thick haze, the corresponding increase in respiratory diseases. In this paper, through the elaboration of fog and thick haze on the body to explore the preventive measures of Chinese medicine on fog and thick haze. The fog and thick haze is a type of heavy air pollution with the composition of the dense fog and dusthaze [1]. Fog and thick haze in the city, especially in the haze of organic pollutants, complex composition, small diameter, can be inhaled and deposited directly in the lungs. Due to haze particles has a certain chemical and biological activity, it has the ability to carry germs, so, there is a greater threat to human health. How to effectively prevent the harm of fog and haze on the body has already become a hot issue in the whole society.
\end{abstract}

\section{Understanding of Chinese Medicine on Haze.}

Haze is a man-made pollution as the main cause of weather phenomena. Chinese medicine believes that haze is a weather phenomenon with a main feature of air turbidity, gloomy and dark as the main performance, its serious haze. Or when the body's righteousness is insufficient, they could be taken ill. Similar to attack of six exogenous, it is one of the exogenous etiology. Haze also has common characteristics with six exogenous. First, exogenous, six exopathogens caused by the disease, most from the skin, nose and mouth into the mouth, it is evident that the haze from the nose and mouth into and first invasion of the lung. Second, seasonal, spring easily has windy disease, summer with more summer-heat disease, long summer and autumn with more dry disease, autumn with more dry disease, winter with more cold disease, and according to modern research shows that haze often appear in autumn and winter, rare in summer, the most common in December. Third, regional, Northwest with more dry disease, Northeast with more cold disease, Jiangnan with hot and humid, while the haze occurred in the middle and lower reaches of the Yangtze River, North China, Sout hern China, and the local climate and environment are closely related, with a clear regional characteristics [2];

\section{Fog and Thick Haze Harm to the Body.}

\section{Effects on Systema Respiratorium.}

Cough. Fog and thick haze has a significant impact on the respiratory system, especially the submicron particles are easily deposited in the upper, lower respiratory 
tract and alveoli. In the clinic, most of which is manifested in the increase in cough syndrome, and even now produced a special term "fog and thick haze cough".

Nasopharyngitis. Fog and thick haze often caused by multiple nasopharyngitis. The common symptoms: nasal congestion, turbid nasal discharge or burbid nasal discharge, and even purulent blood, pharyngeal discomfort, pharyngeal foreign body sensation, dry throat, sore throat. Combined with the current situation of environmental pollution on the health of the actual situation, the combination of dampness and cold or heat invasion of the lung of the first, leading to the incidence of pharyngitis. Among them, the sensitive population and the high incidence of children [3].

Asthma. Fog and thick haze and other air pollution can also lead to the occurrence and exacerbation of asthma. Air pollutants may cause oxidative stress through the removal of antioxidants, which leads to the damage of airway epithelium, and is involved in the pathogenesis of asthma. Secondly, the air pollutants and the airway wall directly affect the airway structure, which affects the expression of inflammatory mediators and the balance of immunological effects [4]. The increased sensitivity of the respiratory system to allergens. Air pollution can enhance the permeability of the airway epithelium, increasing the contact of airway epithelial and allergen. Children with asthma were more likely to be affected by air pollution in all age groups [5].

Lung cancer. In October 17, 2013, a report by the International Institute for cancer research, the World Health Organization (WHO), for the first time pointed out that air pollution is a cause of cancer in humans and is considered a common and major environmental carcinogen.

\section{Effect on Cardiovascular System}

Fog and thick haze particles can change the intensity of blood vessels, causing atherosclerosis, autonomic nervous response and systemic inflammatory reaction. In the short-term haze environment can induce arrhythmia, myocardial infarction, myocardial ischemia, heart failure, stroke, aggravation of peripheral arterial disease, and sudden death. In the long-term haze environment can increase the risk of high blood pressure and systemic atherosclerosis and other cardiovascular diseases [6]. The clinical symptoms of nose heavy and limb weakness, inappetence, or fever fluctuation, lingering illness, sense of suppression in the chest, abdominal fullness and distention, shortness of breath, fatigue, palpitations, slow heartbeat, pale tongue, white greasy fur, floating and soft pulse or late or slow or intermittent pulse.

\section{Effects on the Reproductive System}

Fog and thick haze, including sulfur dioxide, nitric oxide, heavy metals and other toxic substances. These pollutants may enter the body through the respiratory tract, through the circulatory system to the reproductive system.I n comparison, male testis and spermatogenesis are more sensitive to some heavy metals. Some toxic substances can cause reproductive organs hypoxia, so that sperm motility and quality decline, thereby affecting the normal development of sperm.

\section{Skin Disease}

At present, more and more allergic skin disease, on the one hand is the internal cause, and personal allergic constitution. On the other hand, it is caused by external factors, and is closely related to food and environmental pollution. Allergic skin disease is characterized by itching, chronic, recurrent, and other allergic diseases. 


\section{Ophthalmology}

Fog and thick haze consists of hundreds of atmospheric particulate matter, which are less than $10 \mu \mathrm{m}$ in diameter. Harmful substances can cause discomfort when they invade the eye, and this discomfort is more likely to occur when wearing contact lenses.

\section{Chinese Medicine for the Prevention of Fog and Thick Haze}

\section{Preventive Measure}

First of all, according to different constitutions to adopt different methods to improve the body's disease resistance. Qi deficiency is prone to sweat, recurring colds. Who could use tonifying Qi of the American ginseng, Astragalus membranaceus Decoction and tea. The man showed lung heat and dry stool, dry mouth and throat, and this kind of person can take the Radix Ophiopogonis, rhizoma phragmitis to clearing away the lung-heat. Diet can eat pears, lotus root, drink hydromel. Deficiency of Yang deficiency of lung qi deficiency is often manifested as cold, often accompanied by chronic cough, and such people can usually use ginger, red dates to drink water. Secondly, the timely separation of fog and haze weather. Fog and haze less window, go out wearing masks. Human skin in direct contact with the air haze in time after bathing. Some green plants in the room, or the use of air purifiers, is conducive to the absorption of harmful gases. Finally, choose the right time for exercise. People who have morning exercises should stop outdoor activities. Because every day 6 to 11 points is more serious pollution period. Relatively speaking, the evening air cleaner, exercise habits of the public can exercise time into the evening [7].

\section{Treatment}

Causes of fog and thick haze disease now has two points, one is that due to dryness caused by pyretic toxicity invasion of lung. There is also a view that is due to the combination of wet, muddy and wind, cold and pathogenic heat. The author believes that the complex external evils caused by the combination of wet, muddy and wind, cold and pathogenic heat from mouse and nose enter into the human body. The formation of a variety of "disease" and "syndrome" in appearance, a reversible or irreversible damage to the organs of the human body parts. Diseases caused by the fog and thick haze of lung disease, cardiovascular and cerebrovascular diseases and skin, reproductive system, eye and other diseases.In the treatment of most of the basic principle of strengthening the body resistance to eliminate pathogenic factors. On the basis of improving their immunity, dialectical treatment of different syndromes.

Cough. According to the different etiology, Huang Yaosheng according to the type of cold, heat and wet to treatment of haze cough. On the basis of Jiaweizhisousan, the cold type was added with Perilla leaf and roasted Ephedra, and if serious, adding ginger, cassia twig. On the basis of Jiaweizhisousan, the heat type was added with mulberry leaf and chrysanthemum, and if serious, adding Scutellaria baicalensis and fructus arctii. On the basis of Jiaweizhisousan, the wet type adds Job's-tears, rhizoma atractylodis, if serious, adding Rhizoma Pinelliae and Amomum tsao-ko. The results showed that the total effective rate was $92 \%$ [8].

Nasopharyngitis. Rhinitis is often treated with percolating the Lung to opening the orifices, ventilation and turbidity. The commonly used single Chinese herbs with Centipeda minima, duckweed, stir fried Fructus Xanthii, herba asari cum radice, ephedra, asarum, Ligusticum wallichii, Magnolia liliflora, rhizoma acori graminei,radix angelicae, etc. The compound to Compound 
Xinyi powder, Xiao Qinglong Tang as the representative. Pharyngitis according to clinical manifestations are divided into the type of cold and heat. Cold symptoms with dry throat, sore throat, phlegm thinning, and chills, fever, headache, pale tongue, thin white fur, floating and tight pulse. General use of warm acrid exterior-resolving medicinal, plus a small amount of cold medicine to clear the throat. The commonly used single Chinese herbs with radix sileris, peppermint, folium perillae, ginger, cordate houttuynia, Scutellaria baicalensis, Platycodon grandiflorum, Radix liquiritiae. The compound particles represented by Compound Herba Houttuyniae. Wind-heat the symptoms have throat, searing pain, dysphagia, phlegm, and fever, headache, thirst, tongue red, moss thin yellow, superficial and rapid pulse. General use of cool acrid exterior-resolving medicinal, the evacuation of wind heat, diffusing the lung and relieve the sore throat mainly. The commonly used herbs with honeysuckle, fructus forsythiae, Arctium, peppermint, radix scrophulariae, Scutellaria baicalensis, rhizoma phragmitis etc.The compound in Yinjushaoyaotang, Maidonglugenyin represented.

Asthma. Clinical treatment methods mainly use anti infection, antispasmodic, desensitization and other methods to control asthma. Modern pharmacological studies show that the effect of silkworm with anti allergic, anti infection, antitussive, antispasmodic. Earthworm has the function of anti histamine and dilating bronchial smooth muscle. Periostracum cicada has sedative and anti allergy, reduce stress, relieve bronchial spasm, muscle blockade of sympathetic ganglia conduction. The treatment of asthma is not only to relieve cough and asthma, but also is tonifying lung and kidney, so that it can relieve asthma. The commonly used single Chinese herbs have Radix Pseudostellariae, Cordyceps sinensis, Fritillaria roylei Hooker, etc. The compound of Compound Radix Pseudostellariae antitussive powder, compound tablet of [Sichuan] fritillary bulb as the representative.

Lung cancer. Chinese medicine believes that lung cancer is a kind of systemic disease, which is characterized by asthenia in origin and asthenia in superficiality. Yin deficiency due to deficiency of vital energy is the essence of lung cancer. Evil seizes the opportunity to invade the meridians, organs, which can cause stagnation of vital energy and blood stasis, phlegm and blood stasis. The phlegm and blood stasis in the lung collaterals. So the treatment to grasp the "strengthening the body resistance", "eliminating evil" balance is especially important. Tonifying spleen and stomach in order to strengthening the body resistance. commonly used in the spleen of a single taste of traditional Chinese medicine, Radix Codonopsis, Atractylodes, Poria, astragalus, etc., and its compound to Decoction of Four Mild Drugs, Zhenqi Fuzheng Granule Effect represented. In the aspect of eliminating evil, Professor Chen Baixian believes that cancer is due to blood stasis retention in the body. Therefore, Arrowhead mountain, Prunella vulgaris and other drug for attacking toxin and resolving stasis based in Xuefu Zhuyu Decoction. According to the condition and then supplemented with heat clearing and detoxifying, soft and hard scattered medicine. Selected as appropriate, Herba Selaginellae Doederleinii, salvia chinensis, Herba Hedyotis, Sculellaria barbata, fruticose dracaena leaf and other drugs of clearing away heat and toxic material ${ }^{[9]}$.

CCVD. The treatment should be eliminated dampness and activated Yang, available Huolian Decoction (Agastache rugosus, Coptis chinensis, Mangnolia officinalis, rhizoma atractylodis, capillary artemisia, cassia twig, Astragalus mongholicus, Ligusticum wallichii, Radix liquiritiae) adding linggui zhugan decoction.

Diseases of Reproductive System. The reproductive system disease caused by fog 
and thick haze belongs to the syndrome of accumulated damp-heat. In the application of a large number of drugs of clearing away heat and promoting diuresis at the same time, also with tonifying Qi, promoting blood circulation for removing blood stasis, tonifying Yang, suppress evil. It could be used Modified Simiao Pills. The prescription of golden cypress, Rhizoma Dioscoreae hypoglaueae, cogongrass rhizome, Poria cocos, psyllium seed,rhizoma alismatis, could clear away heat and promoting diuresis, and nourishing yin and cooling blood. Radices cyathulae, Lulutong could promoting qi circulation and removing blood stasis. Atractylodes macrocephala and herba cistanches could tonify Yang and strengthen the body resistance. In addition, from the psychology, fog and thick haze will give people a dull and depressing feeling, stimulating mental depression, and treatment available changpuyujintang and YueJuWan ${ }^{[10]}$.

Skin Disease. Chinese medicine believes that the disease due to the individual quality being not strong, poor tolerance, and contact with some of the "feeling" and the evil invasion of the skin, mucous membrane, and then heat, heat and blood stasis. In the course of treatment, syndrome differentiation and treatment of wind heat syndrome is best to use the method of clearing heat and dispelling wind, with the addition of Xiaofengsan. Heat syndrome should be cleaned away heat and promoting diuresis, with Longdan Xiegan Tang. Syndrome of heat in the blood system should be removed pattogenic heat from the blood and toxic material from the body. Of course, such as fangfeng, Schizonepeta, duckweed, periostracum cicada, cortex dictam all have anti allergic effects.

Keratoconjunctivitis Sicca. For mild eye discomfort, using Chinese medicine fumigation eyes of the method is relatively simple and practical. There are many common traditional Chinese medicine can play the role of improving eyesight, such as chrysanthemum clearing liver and improving vision, Medlar tonifying kidney for improve eyesight, semen cassiae torae Improving eyesight and defecation, green tea removing heat to brighten vision. This is more beneficial to fumigate eyes with Chinese herbal medicine and green tea on the weather of fog and thick haze ${ }^{[11]}$.

\section{Conclusion}

Fog and thick haze weather can cause people to the respiratory system, cardiovascular system diseases, reducing the body's immune function, affected fertility and mental health. The use of appropriate care methods and traditional Chinese medicine health care, could effectively fight fog and thick haze on the impact of people. For the fog and thick haze impact, focusing on prevention. In short, according to fog and thick haze caused by human heart and lung function damage and its clinical manifestations, we recognize that fog and thick haze belongs to exogenous evil, and everyone can be ill for that, but it without infectiousness. It from the nose and mouth into the lungs, and then heart, Qi, blood. The prevention of fog and thick haze is mainly strengthening body resistance and eliminating evil. The righteous memory, do not be evil. Fog and thick haze causes disease, damage being light, if serious could cause disease, which seriously harm to human health. For the fog and thick haze caused by disease related issues still need to conduct a comprehensive and in-depth study, to carry out animal experimental studies, and found that different components caused by different pathological damage. There are already some applications of urban fog particles, nitrogen dioxide and other harmful gases for animal experiments, indicating that fog and thick haze can induce oxidative stress and induce promotion inflammation cytokine expression. Therefore, it is helpful for us to make effective 
prevention and cure measures to clarify the pathological damage characteristics of fog and thick haze.

\section{Acknowledgements}

The research work was supported by National Science and technology letter (2016) No. 65, Zhongyuan scholar(162101510003), Henan excellent science and technology innovation team(TCJ2014-391).

\section{Reference}

[1]Fang XU. Some thoughts on the prevention and cure of fog and thick haze[J].Journal of Shanxi provincial Party School,2014,19-20.“In Chinese"

[2]Ya-qin ZHANG, Zhou-ping SHEN, Ye CHEN. Application of TCM Health Management in the prevention of respiratory diseases of susceptible population in foggy and hazy season[J]. Health Research, 2016, 36 (5):493-495."In Chinese"

[3] Yang JIAO, Zhi-song WU, Fang CAO. Characteristics of Etiology and Pathogenesis in Fog and Haze Causing Diseases [J].Journal of Traditional Chinese Medicine, 2016,57(9):740-742."In Chinese"

[4]CHEN Y,EBENSTEIN A,GREENSTONE M,et al. Evi-dence on the impact of sustained exposure to air pollutionon life expectancy from China's Huai River policy [J].Proc Natl Acad Sci USA,2013,110( 32) : 12936-12941.

[5]RAASCHOU-NIELSEN O, ANDERSEN ZJ, JENSENSS,et al. Traffic air pollution and mortality from cardio-vascular disease and all causes: a Danish cohort study [J]. Environ Health, 2012, 11(20): 60-71.

[6] Lin XING, Wei WANG, Yang YU,et al. Influence of hazy weather on respiratory and cardiovascular diseases in the elderly[J]. Occup and Health, 2014, 30 (16): 2277 2279.“"In Chinese"

[7] Chou-fu CHENG. TCM Cognition and Treatment Thought to the Disease Caused by the Haze [J]. Journal of Hunan Univ. of CM, 2014,34 (5):1-4. "In Chinese" [8]Yaosheng HUANG . Observation on therapeutic effect of modified Zhike powder in treating cough caused by fog and thick haze [J]. Journal of Zhejiang University of Traditional Chinese Medicine, 2014,38 (6):730-732."In Chinese"

[9] Yao-shen HUANG. "Haze Cough" Treated with Revised Zhi-sou Powder(with Comparative Report of 145 Cases) [J].Hunan Journal of Traditional Chinese Medicine, 2015,31(11):142-143.“In Chinese”

[10]Li-zhen ZHAO, Yao-qun ZHU, Chen-liang He, et al. Clinical observation of 60 cases of two eyes of winter Yuzhu decoction combined treatment with artificial tears dry[J].Zhejiang Journal of Traditional Chinese Medicine, 2012,47( 5) :345-346."In Chinese"

[11] Ming PAN. Effects of fog and thick haze on human health [J].Studies of Trace Elements and Heal, 2013, 30 (5):65-66."In Chinese” 